\title{
Fast transitionless expansion of cold atoms in optical Gaussian-beam traps
}

\author{
E. Torrontegui, ${ }^{1}$ Xi Chen, ${ }^{1,2}$ M. Modugno, ${ }^{3,4}$ A. Ruschhaupt,${ }^{5}$ D. Guéry-Odelin, ${ }^{6}$ and J. G. Muga ${ }^{1,7}$ \\ ${ }^{1}$ Departamento de Química Física, Universidad del País Vasco-Euskal Herriko Unibertsitatea UPV/EHU, Apartado 644, Bilbao, Spain \\ ${ }^{2}$ Department of Physics, Shanghai University, 200444 Shanghai, People's Republic of China \\ ${ }^{3}$ Departamento de Física Teórica e Historia de la Ciencia, Universidad del País Vasco-Euskal Herriko Unibertsitatea UPV/EHU, \\ Apartado 644, Bilbao, Spain \\ ${ }^{4}$ IKERBASQUE, Basque Foundation for Science, Bilbao, Spain \\ ${ }^{5}$ Institut für Theoretische Physik, Leibniz Universität Hannover, Appelstraße 2, 30167 Hannover, Germany \\ ${ }^{6}$ Laboratoire Collisions Agrégats Réactivité, CNRS UMR 5589, IRSAMC, Université Paul Sabatier, 118 Route de Narbonne, \\ 31062 Toulouse CEDEX 4, France \\ ${ }^{7}$ Max Planck Institute for the Physics of Complex Systems, Nöthnitzer Straße, Dresden, Germany
}

(Received 31 October 2011; published 5 March 2012)

\begin{abstract}
We study fast expansions of cold atoms in a three-dimensional Gaussian-beam optical trap. Three different methods to avoid final motional excitation are compared: inverse engineering using Lewis-Riesenfeld invariants, which provides the best overall performance, a bang-bang approach, and a fast adiabatic approach. We analyze the excitation effect of anharmonic terms, radial-longitudinal coupling, and radial-frequency mismatch. In the inverse-engineering approach these perturbations can be suppressed or mitigated by increasing the laser beam waist.
\end{abstract}

DOI: 10.1103/PhysRevA.85.033605

PACS number(s): 03.75.-b, 03.65.Nk

\section{INTRODUCTION: DRIVEN EXPANSIONS OF COLD ATOMS}

Driving the expansion of a Bose-Einstein condensate or, more generally, of a cold atom cloud in a controlled way by implementing a designed time dependence of the trap frequency is a common and basic operation in a cold-atom laboratory. The expansion may be aimed at different goals, such as decreasing the temperature [1,2], adjusting the density to avoid three-body losses [3], facilitating temperature and density measurements [3], or changing the size of the cloud for further manipulations [4]. Expansions, isolated [5] or as part of refrigeration cycles [6], are also important from a fundamental point of view to quantify the third principle of thermodynamics. ${ }^{1}$ In general, changes of the confining trap will excite the state of motion of the atoms unless they are done very slowly or, in the usual quantum-mechanical sense of the word, "adiabatically," but slow-change processes are also prone to perturbations and decoherence or are impractical for performing many cycles. Engineering fast expansions without final excitation is thus receiving much attention recently, both theoretically and experimentally [5-22]. Most theoretical treatments so far are for idealized one-dimensional (1D) systems, but the implementation requires a three-dimensional (3D) trap $[11,14,15]$, in principle with anharmonicities and couplings among different directions.

In this paper we address these important aspects to implement the expansion in practice. Specifically, we shall model a simple physical realization based on an elongated cigar-shaped optical dipole trap with cylindrical symmetry; see Fig. 1. Far-off-resonance optical confinement has many advantages: it enables the manipulation of spinor condensate

\footnotetext{
${ }^{1}$ Compressions may also play a role in many experiments, but their treatment is similar to expansions, so we shall not deal with them here explicitly.
}

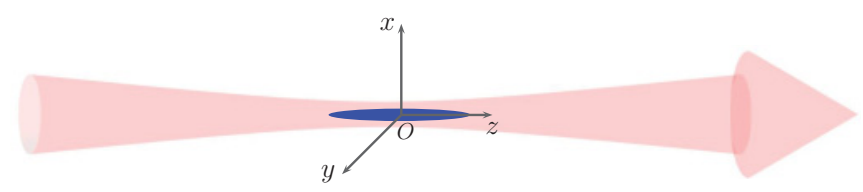

FIG. 1. (Color online) Schematic view of the optical trap.

$[23,24]$ and can be applied to atoms with no permanent magnetic moment [25], which is particularly interesting for metrology. The optical trap in this paper is formed by a single laser that is red detuned with respect to an atomic transition to make the potential attractive (Sec. II) and is characterized in the harmonic approximation by longitudinal and radial frequencies. While magnetic traps allow for an independent control of longitudinal and radial frequencies [11,14,15], this is not the case for a simple laser trap that therefore requires a special study. We assume that the time dependence of the longitudinal frequency is engineered to avoid final excitations with a simple 1D harmonic theory (Sec. III) and analyze the final fidelity in the actual trap. Even though for full 3D results we resort to a purely numerical calculation in Sec. V, an understanding of the effects involved is achieved first by analyzing separately longitudinal and radial motions in Sec. IV. Conclusions and open questions are drawn in Sec. VI.

\section{THE MODEL}

The intensity profile of a Gaussian laser beam in the paraxial approximation is given by

$$
I(r, z, t)=I_{0}(t) e^{-2 r^{2} / w^{2}(z)} \frac{1}{1+z^{2} / z_{R}^{2}},
$$

where $r$ and $z$ are the radial and longitudinal coordinates, respectively, and the variation of the spot size $w$ with $z$ is 
given by

$$
w(z)=w_{0} \sqrt{1+\left(\frac{z}{z_{R}}\right)^{2}},
$$

where $z_{R}=\pi w_{0}^{2} / \lambda$ is the Rayleigh range, $w_{0}$ is the waist, and $\lambda$ is the laser wavelength. The paraxial approximation is valid for waists larger than about $2 \lambda / \pi$.

If an atom is placed in this field with a detuning $\delta$ that is large with respect to the Rabi frequency $\Omega$ and the inverse of the lifetime of the excited state $\Gamma=\tau^{-1}$ but small with respect to the transition frequency, internal excitations and counterrotating terms are negligible, and the potential that the ground-state atoms feel due to the dipole force is proportional to the laser intensity,

$$
V(r, z, t) \simeq \frac{\hbar \Omega^{2}}{4 \delta}=\frac{\hbar \Gamma}{8} \frac{\Gamma}{\delta} \frac{I}{I_{\mathrm{sat}}}, \quad|\delta| \gg \Omega, \Gamma,
$$

and inversely proportional to $\delta . I_{\text {sat }}=\pi h c /\left(3 \lambda^{3} \tau\right)$ is the saturation intensity. ${ }^{2}$ Combining Eqs. (1) and (2) and adding for convenience in later expressions the physically irrelevant term $V_{0}(t)$, the potential takes finally the form

$$
V(r, z, t)=-V_{0}(t) e^{-2 r^{2} / w^{2}(z)} \frac{1}{1+z^{2} / z_{R}^{2}}+V_{0}(t),
$$

where $V_{0}(t)=I_{0}(t) \hbar \Gamma^{2} /\left(8 \delta I_{\text {sat }}\right)$. The analysis carried out here is not restricted to a two-level atom. Indeed, the dipole force is always proportional to the intensity for a multilevel atom but with a coefficient that depends on the level structure and the light polarization [26]. As an example of the magnitudes involved in practice, for rubidium- 87 atoms, a Gaussian beam linearly polarized with a waist of $8 \mu \mathrm{m}$, power $P=30 \mathrm{~W}$, and a wavelength of $1060 \mathrm{~nm}$ provides a longitudinal trap frequency $\omega_{0 z} / 2 \pi \simeq 2500 \mathrm{~Hz}$. We shall use these or similar values in numerical examples below.

In this work we shall assume small enough densities and times so that a one-particle Schrödinger description for the atomic motion neglecting collisions is valid. Extensions to Tonks-Girardeau gases or condensates are possible as in $[7,8,14]$. We shall also assume that the longitudinal axis lies horizontally and neglect the effect of gravity, either because it is artificially compensated or because the radial confinement is tight. ${ }^{3}$

To solve the time-dependent Schrödinger equation associated with the potential in Eq. (3),

$$
i \hbar \frac{\partial \Psi}{\partial t}=-\frac{\hbar^{2}}{2 m} \nabla^{2} \Psi+V \Psi
$$

\footnotetext{
${ }^{2}$ Indeed, other regimes could be used as well [26]. For larger detunings counterrotating terms become important, but the dipole potential is still proportional to the laser intensity. In the extreme limit of quasielectrostatic traps the frequency of the trapping light is much smaller than the resonance frequency, precluding the possibility of a potential sign change from attractive to repulsive; this change is, in principle, allowed by Eq. (2) by changing the sign of the detuning across resonance, but see the discussion below.

${ }^{3}$ This requires that the vertical shift of the trap center in the gravity field is small with respect to the waist, or $g \ll w_{0} \omega_{R}^{2}$, where $\omega_{R}$ is the radial angular frequency and $g$ is the acceleration due of gravity.
}

we use cylindrical coordinates,

$$
\nabla^{2} \equiv \frac{1}{r} \frac{\partial}{\partial r}\left(r \frac{\partial}{\partial r}\right)+\frac{1}{r^{2}} \frac{\partial^{2}}{\partial \phi^{2}}+\frac{\partial^{2}}{\partial z^{2}} .
$$

With the standard separation of variables $\Psi=\mathcal{F}(r, z, t) \Phi(\phi)$, we find

$$
-\frac{\hbar^{2}}{2 m}\left[\frac{1}{r}\left(\frac{\mathcal{F}_{r}}{\mathcal{F}}+r \frac{\mathcal{F}_{r r}}{\mathcal{F}}\right)+\frac{1}{r^{2}} \frac{\Phi_{\phi \phi}}{\Phi}+\frac{\mathcal{F}_{z z}}{\mathcal{F}}\right]+V=i \hbar \frac{\mathcal{F}_{t}}{\mathcal{F}}
$$

(the simple and double subscripts denote first and second derivatives, respectively), whereas the equation for $\Phi$ reads

$$
\frac{\Phi_{\phi \phi}}{\Phi}=-v^{2} \quad v \in \mathbb{Z}
$$

where $v$ is a magnetic quantum number determining the conserved angular momentum component along the $z$ direction. This is solved by $e^{i v \phi}$, so we shall concentrate on $\mathcal{F}(r, z, t)$. Defining now $\tilde{\Psi}(r, z, t)=\sqrt{r} \mathcal{F}(r, z, t)$, we get

$$
i \hbar \frac{\partial \tilde{\Psi}}{\partial t}=-\frac{\hbar^{2}}{2 m} \tilde{\nabla} \tilde{\nabla}^{2} \tilde{\Psi} \tilde{V} \tilde{\Psi}
$$

where

$$
\begin{gathered}
\tilde{\nabla}^{2} \equiv \frac{\partial^{2}}{\partial r^{2}}+\frac{\partial^{2}}{\partial z^{2}}, \\
\tilde{V} \equiv \frac{\hbar^{2}}{2 m}\left(\frac{v^{2}-1 / 4}{r^{2}}\right)+V(r, z, t),
\end{gathered}
$$

and $V(r, z, t)$ is given by Eq. (3). The task is now to design $V_{0}(t)$ so as to achieve a fast expansion without final excitation.

\section{EXPANSION PROTOCOLS}

In this section we briefly present three expansion protocols for a one-dimensional harmonic trap. They are chosen mostly because they have been realized experimentally [1,11,14,27]. Their simplicity will help us to identify relevant physical phenomena that will affect also other protocols. No claim of completeness or global optimization is made, and there is certainly room for investigating other protocols.

The objective is to expand the trap from an initial frequency $\omega_{0} / 2 \pi$ to a final frequency $\omega_{f} / 2 \pi$ in a time $t_{f}$, without inducing any final excitation in the adiabatic or instantaneous basis for which the Hamiltonian is diagonal. Note that transient excitations are generally allowed.

\section{A. Inverse engineering with Lewis-Riesenfeld invariants}

Lewis-Riesenfeld invariants may be used to engineer efficient expansion protocols as explained in [5,7,8,11]. We refer the reader to these works for the details and give here only the elements required for a practical application. For any harmonic oscillator expansion and, in fact, for any potential with the structure

$$
V(q, t)=\frac{m}{2} \omega^{2}(t) q^{2}+\frac{1}{b^{2}(t)} U\left(\frac{q}{b(t)}\right),
$$

where the function $U$ is arbitrary, there is a quadratic-inmomentum invariant operator of the form

$$
\begin{aligned}
\mathcal{I}(q, p, t)= & \frac{1}{2 m}\left[b^{2} p^{2}-m \dot{b} b(q p+p q)+m^{2} \dot{b}^{2} q^{2}\right] \\
& +\frac{1}{2} m \omega_{0}^{2}\left(\frac{q}{b}\right)^{2}+U\left(\frac{q}{b}\right),
\end{aligned}
$$


where $q$ and $p$ are generic position and momentum operators (particularized later for longitudinal or radial directions) and $b=b(t)$ is a scaling function that satisfies the Ermakov equation

$$
\ddot{b}+\omega^{2}(t) b=\omega_{0}^{2} / b^{3} .
$$

To inverse engineer the trap frequency, $b(t)$ is designed to satisfy the boundary conditions that guarantee no final excitations and continuity of the trap frequency,

$$
\begin{array}{lll}
b(0)=1, & \dot{b}(0)=0, & \ddot{b}(0)=0, \\
b\left(t_{f}\right)=\gamma, & \dot{b}\left(t_{f}\right)=0, & \ddot{b}\left(t_{f}\right)=0,
\end{array}
$$

where $\gamma=\sqrt{\omega_{0} / \omega_{f}}$. Finally, $\omega(t)$ is deduced from Eq. (11). When these conditions are satisfied, the $n$th eigenstate of $H(0)=p^{2} /(2 m)+V(q, 0)$ evolves as an "expanding mode," which is the time-dependent $n$th eigenvector of the invariant times a "Lewis-Riesenfeld" phase factor $e^{i \alpha_{n}}$, where $\alpha_{n}(t)=$ $-(n+1 / 2) \omega_{0} \int_{0}^{t} d t^{\prime} / b^{2}$, until it becomes an $n$th eigenvector of the final Hamiltonian $H\left(t_{f}\right)=p^{2} /(2 m)+V\left(q, t_{f}\right)$.

Discontinuities in $\ddot{b}$ may, in principle, be allowed, but they amount to performing finite jumps of the trap frequency. This is an idealization, but it can be approached up to technical limits. Discontinuities in $\dot{b}$ have more serious consequences, as they imply $\delta$ functions for $\ddot{b}$ and infinite trap frequencies at the jumps. Thus their approximate physical realization is even more difficult, but they are useful for finding bounds for physical variables of interest, as in [5] or in Appendix A. There are many $b(t)$ that satisfy Eq. (12). A simple smooth function is a quintic polynomial with six coefficients,

$$
b(t)=6(\gamma-1) s^{5}-15(\gamma-1) s^{4}+10(\gamma-1) s^{3}+1,
$$

where $s:=t / t_{f}$. More sophisticated choices are possible to minimize the process time, the average energy, or other variables with or without imposed constraints ("bounded control") on the allowed trap frequencies [12,28].

In principle this method allows for arbitrarily small values of $t_{f}$, but for very small process times the potential becomes transitorily repulsive [8]. With a laser beam, a repulsive potential could be achieved by means of a positive (blue) detuning. However, the validity of Eq. (3) requires a large detuning, so care should be exercised when crossing the resonance. In this work the numerical examples are restricted to positive trap frequencies since the transient passage from attractive to a repulsive interaction could involve unwanted effects such as radiation pressure. We shall, in any case, point out in the figures the minimum value of $t_{f}$ for which the potential stays attractive for all times with the quintic $b(t)$.

\section{B. Bang bang}

Bang-bang methods are those with a stepwise constant behavior of some variable. The simplest one to avoid final excitations consists of using a constant intermediate trap frequency, the geometric average of the initial and final frequencies,

$$
\omega^{\text {bang }}= \begin{cases}\omega_{0}, & t \leqslant 0, \\ \left(\omega_{0} \omega_{f}\right)^{1 / 2}, & 0<t<t_{f}^{\text {bang }} \\ \omega_{f}, & t \geqslant t_{f}^{\text {bang }}=\pi /\left[2\left(\omega_{0} \omega_{f}\right)^{1 / 2}\right],\end{cases}
$$

during a fourth of the corresponding period. Optimal bangbang trajectories with two intermediate frequencies and arbitrary final times are also possible [6,8], but they are not considered here. To arrive at Eq. (14), one may use a classical argument [27] or proceed as follows: the solution of the Ermakov equation for $t>0$ assuming a constant intermediate angular frequency $\omega_{1}$ with initial boundary conditions $b(0)=$ $1, \dot{b}(0)=0$ is

$$
b(t)=\sqrt{\left[\left(\omega_{0}^{2}-\omega_{1}^{2}\right) / \omega_{1}^{2}\right] \sin ^{2}\left(\omega_{1} t\right)+1} .
$$

Then, if we solve for $t_{f}$ and $\omega_{1}$ the two equations implied by the final boundary conditions,

$$
b\left(t_{f}\right)=\gamma, \dot{b}\left(t_{f}\right)=0,
$$

the values in Eq. (14) are found.

\section{Fast adiabatic protocols}

"Fast" and "adiabatic" may appear to be contradictory concepts. A fast adiabatic protocol seeks to perform a process as quickly as possible while keeping it adiabatic at all times. For harmonic oscillator expansions the adiabaticity condition reads $\dot{\omega} / \omega^{2} \ll 1$, so making $\dot{\omega} / \omega^{2}$ constant from $t=0$ to $t_{f}[1]$ and solving the resulting differential equation for $\omega(t)$, we get [8]

$$
\omega^{a d i}(t)=\frac{\omega_{0}}{1-\left(\omega_{f}-\omega_{0}\right) \frac{t}{t_{f} \omega_{f}}} .
$$

\section{LONGITUDINAL AND RADIAL MOTIONS}

By expanding the potential in Eq. (3) in a double Taylor series around $(z=0, r=0)$,

$$
\begin{aligned}
& V(r, z, t) \\
& \simeq-V_{0}(t)\left(-\frac{2 r^{2}}{w_{0}^{2}}-\frac{z^{2}}{z_{R}^{2}}+\frac{2 r^{4}}{w_{0}^{4}}+\frac{z^{4}}{z_{R}^{4}}+\frac{4 r^{2} z^{2}}{w_{0}^{2} z_{R}^{2}}+\cdots\right),
\end{aligned}
$$

we see that the first coupling term between radial and longitudinal motions is of fourth order, proportional to $r^{2} z^{2}$. If we could neglect this and higher-order coupling terms, longitudinal and radial motions would be independent. The approximation that considers longitudinal and radial motions to be completely uncoupled is useful to analyze several effects separately and gain insight. Not only that, as we shall confirm later with full 3D calculations, this is also a good approximation numerically for low-energy levels.

\section{A. Longitudinal motion}

To study the motion in the longitudinal direction we consider first the full longitudinal Hamiltonian [putting $r=0$ in Eq. (3)]

$$
H(z, t)=-\frac{\hbar^{2}}{2 m} \frac{\partial^{2}}{\partial z^{2}}-V_{0}(t)\left(\frac{1}{1+z^{2} / z_{R}^{2}}-1\right) .
$$


To characterize this potential in terms of a longitudinal frequency consider the harmonic approximation

$$
\begin{aligned}
H_{\mathrm{har}}(z, t) & =-\frac{\hbar^{2}}{2 m} \frac{\partial^{2}}{\partial z^{2}}+V_{0}(t) \frac{z^{2}}{z_{R}^{2}} \\
& =\frac{p_{z}^{2}}{2 m}+\frac{m \omega_{z}^{2}(t) z^{2}}{2},
\end{aligned}
$$

where $p_{z}=-i \hbar \partial / \partial z$ and

$$
\omega_{z}^{2}(t)=\frac{2 V_{0}(t)}{m z_{R}^{2}} .
$$

We may now apply the methods described in Sec. III. We shall add a subscript $z$, for longitudinal, to the trap frequencies and take $q \rightarrow z$. For an imposed $\omega_{z}(t)$ and fixed waist and laser frequency, Eq. (21) determines the time dependence of the laser intensity.

Figure 2 shows for the ground state, $n=0$, the "longitudinal fidelity" $F_{L}=\left|\left\langle Z_{n}\left(t_{f}\right)\left|U_{z}\left(t_{f}, 0\right)\right| Z_{n}(0)\right\rangle\right|$, where $Z_{n}(z, 0)$ and $Z_{n}\left(z, t_{f}\right)$ are the initial and final $n$th eigenstates of the full longitudinal Hamiltonian, Eq. (19), for frequencies $\omega_{0 z}$ and $\omega_{f z}$, respectively, and $U_{z}\left(t_{f}, 0\right)$ is the evolution operator with Eq. (19). As in all figures hereafter the initial longitudinal frequency of the trap is $\omega_{0 z} / 2 \pi=2500 \mathrm{~Hz}$, and the wave-
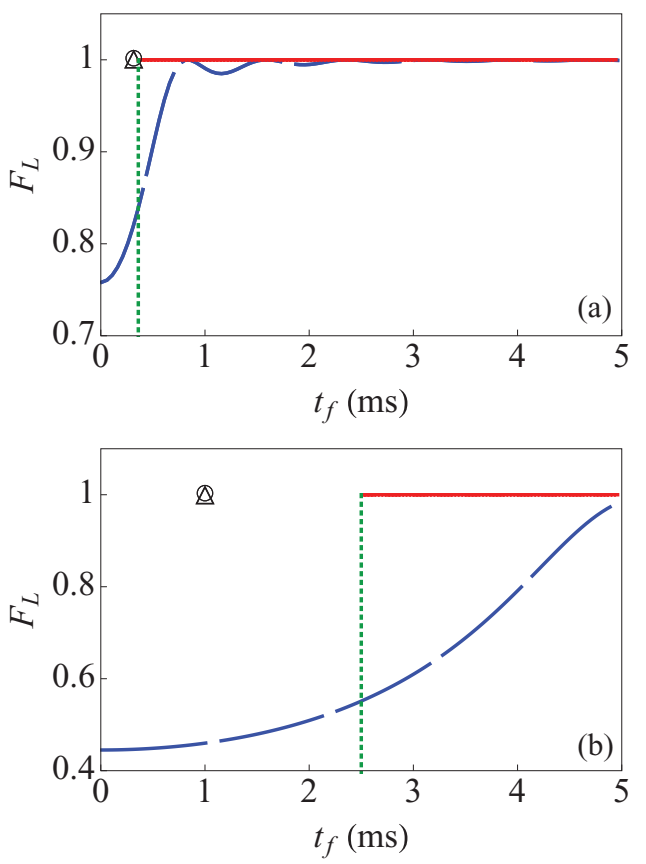

FIG. 2. (Color online) Longitudinal fidelity for Eq. (19) versus final time $t_{f}$ for two different final frequencies: in (a) $\omega_{f z} / 2 \pi=$ $250 \mathrm{~Hz}$; in (b) $\omega_{f z} / 2 \pi=25 \mathrm{~Hz}$. In both plots $\omega_{0 z} / 2 \pi=2500 \mathrm{~Hz}$, $\lambda=1060 \mathrm{~nm}$, and the initial state is the ground state. All curves have been calculated for two different waists, $w_{0}=10 \mu \mathrm{m}$ and $w_{0}=3 \mu \mathrm{m}$, but the results are indistinguishable. $V_{0}(t)$ is chosen according to the three protocols: inverse engineering, using Eq. (13) in Eq. (11) (solid red line), bang-bang (circles for $w_{0}=10 \mu \mathrm{m}$ and triangles for $w_{0}=3 \mu \mathrm{m}$ ), and the fast adiabatic method (long-dashed blue line). The green vertical lines denote the minimum $t_{f}$ for which $V_{0}(t)$ remains positive for all $t$ in the inverse-engineering approach with a quintic $b(t)$. length is taken as $\lambda=1060 \mathrm{~nm}$, which is characteristic of Neodymium-doped lasers. The vertical green lines in Fig. 2 and the following figures correspond to the minimum $t_{f}$ value for which $\omega_{z}(t)$ is positive for all $t$ using the quintic $b(t)$. Of course this limit only applies to the inverse engineering approach.

The three methods are compared for two different final frequencies [in Fig. 2(a) $\omega_{f z} / 2 \pi=250 \mathrm{~Hz}$ and in Fig. 2(b) $\left.\omega_{f z} / 2 \pi=25 \mathrm{~Hz}\right]$ and two different waists; see the caption for details. Actually, the effect of the waist change is negligible in the scale of Fig. 2. Globally, the inverse-engineering method outperforms the others. The bang-bang approach provides a good fidelity, but only for a specific final time, and the fast adiabatic method fails, in fact, to be adiabatic at short times. This is very evident for the smaller final frequency in Fig. 2(b): the opening of the trap is faster and the level spacings are smaller than for the larger final frequency, so the state cannot follow an adiabatic behavior.

For small $t_{f}$ and/or large $\gamma$ the transient excitation energy during the inverse-engineering protocol may be high, giving the atoms access to the anharmonic part of the potential. This could lead to a decay of fidelity as $t_{f}$ decreases, but the effect is not seen in the scale of Fig. 2. The small effect of anharmonicity is enhanced by increasing the vibrational number; see Fig. 3. It can be avoided by increasing the waist, which reduces the anharmonic terms, as demonstrated in Fig. 3. Appendix A provides a perturbation theory analysis of longitudinal anharmonicity. A first-order approach gives analytical lower bounds for the fidelity, and the possibility to maximize them by other choices of $b(t)$. More accurate results are found at second order; see Fig. 3.

\section{B. Radial motion}

To study radial motion we define the radial Hamiltonian by setting $z=0$ in Eq. (3), and we add the "centrifugal term" (an

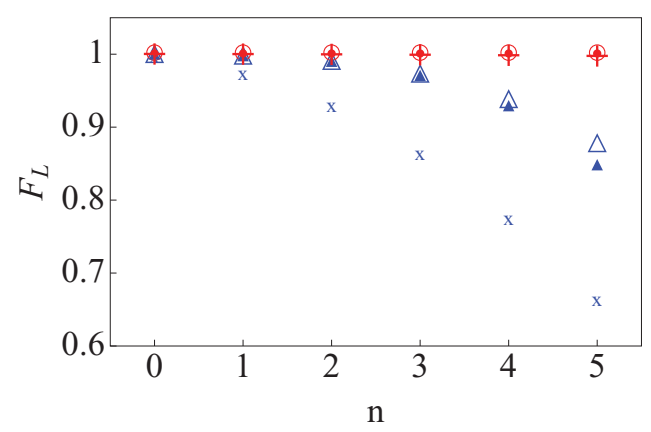

FIG. 3. (Color online) Longitudinal fidelity versus level number $n$. For $w_{0}=3 \mu \mathrm{m}$ [blue (dark gray) symbols], fidelity $F_{L}=$ $\left|\left\langle Z_{n}\left(t_{f}\right)\left|U_{z}\left(t_{f}, 0\right)\right| Z_{n}(0)\right\rangle\right|$ with inverse engineering computed for Eq. (19) and a quintic $b(t)$ (open triangles), first-order bound in Eq. (A9) (crosses), and second-order approximate fidelity computed with Eqs. (A11) and (A12) for the quintic $b(t)$ (solid triangles). For a larger waist, $w_{0}=10 \mu \mathrm{m}$ [red (light gray) symbols], the corresponding values (open circles, pluses, and solid circles) are very nearly 1 and are indistinguishable. In all cases $\omega_{f z} / 2 \pi=25 \mathrm{~Hz}$, $\omega_{0 z} / 2 \pi=2500 \mathrm{~Hz}, \lambda=1060 \mathrm{~nm}$, and $t_{f}=2.5 \mathrm{~ms}$. 
attractive one for $v=0$ ),

$$
\begin{aligned}
H(r, t)= & -\frac{\hbar^{2}}{2 m} \frac{\partial^{2}}{\partial r^{2}}-V_{0}(t)\left(e^{-2 r^{2} / w_{0}^{2}}-1\right) \\
& +\frac{\hbar^{2}}{2 m}\left(\frac{v^{2}-1 / 4}{r^{2}}\right) .
\end{aligned}
$$

In the harmonic approximation we take

$$
-V_{0}(t)\left(e^{-2 r^{2} / w_{0}^{2}}-1\right) \sim \frac{m \omega_{R}^{2}(t) r^{2}}{2},
$$

where

$$
\omega_{R}^{2}(t) \equiv \frac{4 V_{0}(t)}{m w_{0}^{2}}
$$

defines the radial frequency of the trap $\omega_{R}(t) /(2 \pi)$.

We assume that $V_{0}(t)$ is set to satisfy the designed longitudinal expansion according to Eq. (21). Substituting this into Eq. (24) gives the relation between radial and longitudinal frequencies,

$$
\omega_{R}(t)=\frac{\sqrt{2} \pi w_{0}}{\lambda} \omega_{z}(t)
$$

which is key to understanding the behavior of the radial wave function. The waist value $w_{0}=\lambda /(\sqrt{2} \pi)$ would make both frequencies equal, but for such a small waist the paraxial approximation fails, and the present theory could not be applied [29].

Now we define the ground-state "radial fidelity" as $F_{R}=$ $\left|\left\langle\phi_{0}\left(t_{f}\right)\left|U_{R}\left(t_{f}, 0\right)\right| \phi_{0}(0)\right\rangle\right|$, where $U_{R}\left(t_{f}, 0\right)$ is the radial evolution operator for $H(r, t)$ and $\phi_{0}(r, 0)$ and $\phi_{0}\left(r, t_{f}\right)$ are ground states of the initial and final radial traps for Eq. (22), with $V_{0}(t)$ given by Eq. (21). In Fig. 4 the radial fidelity is depicted for the three protocols explained above and $v=0$. The bang-bang trajectory causes much excitation and gives a rather poor

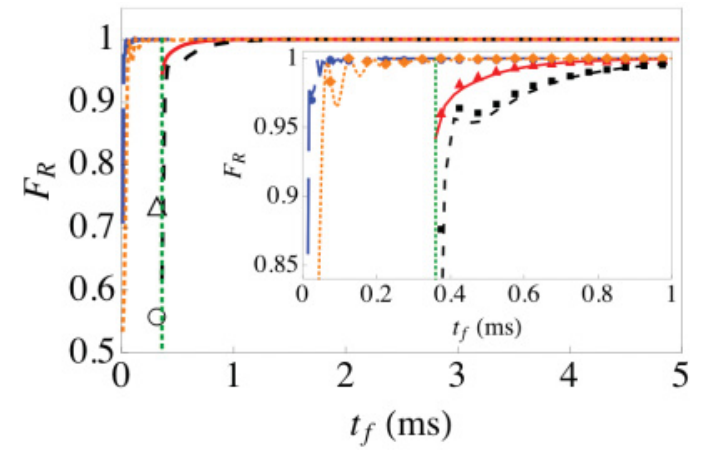

FIG. 4. (Color online) Radial fidelity for different final times. Parameters: $v=0, \omega_{0 z} / 2 \pi=2500 \mathrm{~Hz}, \omega_{f z} / 2 \pi=250 \mathrm{~Hz}$, and $\lambda=1060 \mathrm{~nm}$. Protocols: inverse engineering, quintic $b, w_{0}=10 \mu \mathrm{m}$ (solid red line); inverse engineering, quintic $b, w_{0}=3 \mu \mathrm{m}$ (shortdashed black line); bang bang, $w_{0}=10 \mu \mathrm{m}$ (open circle); bang bang, $w_{0}=3 \mu \mathrm{m}$ (open triangle); fast adiabatic, $w_{0}=10 \mu \mathrm{m}$ (long-dashed blue line); fast adiabatic, $w_{0}=3 \mu \mathrm{m}$ (dotted orange line). In the inset, the corresponding red triangles, black squares, blue circles, and orange diamonds are the approximate fidelities from adiabatic perturbation theory using Eq. (28). The green vertical line near $0.4 \mathrm{~ms}$ is the minimal final time $t_{f}$ for which the quintic- $b$ inverseengineering protocol implies positive frequencies at all transient times. fidelity in the radial direction (see the big open symbols). This is because, even though the "correct" transient radial frequency $\omega_{R}^{\text {bang }}(t)=\left(\sqrt{2} \pi w_{0} / \lambda\right) \omega_{z}^{\text {bang }}(t)$ is implemented (as the geometric average of initial and final radial frequencies), the time $t_{f}$ is adjusted for the longitudinal not for the radial frequency; compare $t_{f z}^{\text {bang }}$ to $t_{f R}^{\text {bang }}:=\lambda t_{f z}^{\text {bang }} /\left(\sqrt{2} \pi w_{0}\right)$.

The behavior of the other two methods is much more robust, as shown by their fidelities in Fig. 4, where the inset amplifies the small- $t_{f}$ region. The fast adiabatic approach (blue long-dashed lines in Fig. 4) gives an excellent radial fidelity. A detailed calculation shows that the adiabaticity condition for the radial Hamiltonian (see Appendix B) takes, in spite of the centrifugal term, the same form as for the ordinary harmonic oscillator, namely, $\dot{\omega}_{R} / \omega_{R}^{2} \ll 1$. If $\dot{\omega}_{z} / \omega_{z}^{2}$ is constant, the ratio $\dot{\omega}_{R} / \omega_{R}^{2}$ will be constant too, but smaller by a factor $\lambda /\left(\sqrt{2} \pi w_{0}\right)$, so that the radial dynamics will be, in general, more adiabatic. Indeed, comparing the blue long-dashed lines in Figs. 2 and 4, we see that the radial fidelity is better than the longitudinal one, as the confinement is tighter radially than longitudinally, and the energy levels are more separated.

For the inverse-engineering protocol with a quintic $b$ (red solid and black short-dashed lines in Fig. 4) the radial excitation is small, the fidelity being nearly 1 for $t_{f}>1$ $\mathrm{ms}$ in the scale of Fig. 4. This could be surprising since the radial frequency does not behave according to an ideal inverse-engineering dependence

$$
\omega_{\text {Rinv }}^{2}(t)=\frac{2 \pi^{2} w_{0}^{2}}{\lambda^{2}} \frac{\omega_{0 z}^{2}}{b^{4}}-\frac{\ddot{b}}{b},
$$

where we have used the Ermakov equation and $\gamma=$ $\left(\omega_{0 z} / \omega_{f z}\right)^{1 / 2}=\left(\omega_{0 R} / \omega_{f R}\right)^{1 / 2}$, so the function $b$ is the same for the longitudinal and radial directions. Instead, the actual radial frequency varies according to Eq. (25),

$$
\omega_{R}^{2}(t)=\frac{2 \pi^{2} w_{0}^{2}}{\lambda^{2}}\left(\frac{\omega_{0 z}^{2}}{b^{4}}-\frac{\ddot{b}}{b}\right) .
$$

The difference between Eqs. (26) and (27) may be quite significant, as the example in Fig. 5 shows, so the radial state does not really follow the expanding modes corresponding to Eq. (26). As a consequence, a perturbative approach based on Eq. (26) as the zeroth order fails completely, even for qualitative guidance. (Further evidence is provided in

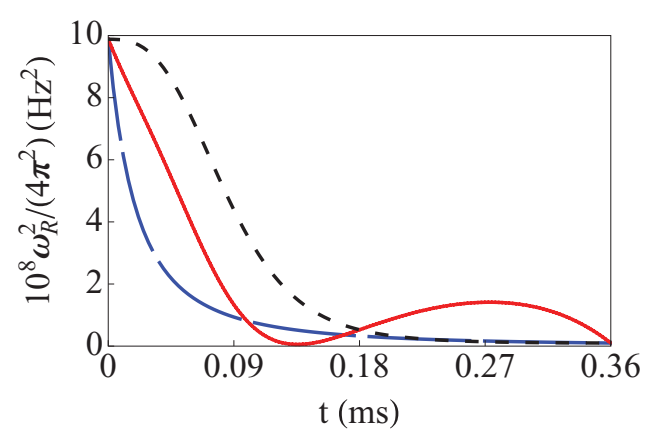

FIG. 5. (Color online) Square of radial frequency vs time: Eq. (27) based on a quintic $b$ (red solid line); fast adiabatic protocol, Eq. (25) with Eq. (17) for $\omega_{z}$ (blue long-dashed line); and Eq. (26) (black short-dashed line). Parameters: $\lambda=1060 \mathrm{~nm}, \omega_{0 z} / 2 \pi=2500 \mathrm{~Hz}$, $\omega_{f z} / 2 \pi=250 \mathrm{~Hz}, w_{0}=3 \mu \mathrm{m}$, and $t_{f}=0.36 \mathrm{~ms}$. 


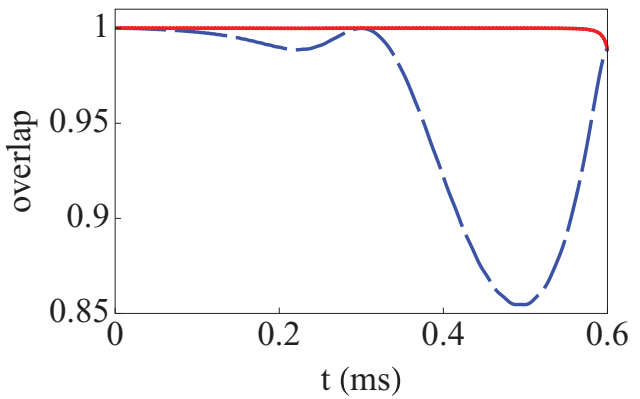

FIG. 6. (Color online) Modulus of the overlap integral between the state evolving from the ground state with the radial Hamiltonian, using the actual radial frequency in Eq. (27) and (i) the instantaneous eigenstate in harmonic approximation (red solid line) or (ii) the expanding mode in harmonic approximation for the radial frequency in Eq. (26) (dashed blue line). Parameters: $v=0, \omega_{0 z} / 2 \pi=2500 \mathrm{~Hz}$, $\lambda=1060 \mathrm{~nm}, \omega_{f z} / 2 \pi=250 \mathrm{~Hz}, w_{0}=3 \mu \mathrm{m}$, and $t_{f}=0.6 \mathrm{~ms}$.

Fig. 6.) Instead, a valid perturbation theory analysis may be based on the zeroth order of the adiabatic modes, which are instantaneous eigenstates of the radial Hamiltonian. The physical reason for the relatively good radial behavior of the invariant-based inverse-engineering protocol is thus the adiabaticity in that direction. When $t_{f}$ approaches the critical lower value, $\omega_{R}(t)$ becomes too steep at small values of $\omega_{R}$ (see Fig. 5), and adiabaticity breaks down, which explains the fidelity decrease there. To mitigate this problem, a larger waist may be used to increase $\omega_{R}(t)$ for a given $\omega_{z}(t)$ [see Eq. (25)], making the radial process more adiabatic and improving the fidelity, as shown in Fig. 4. Keep in mind that to implement a given $\omega_{z}(t)$ an increase of waist has to be compensated by an increase of laser intensity.

Inserting a wave-function expansion in an adiabatic basis (instantaneous eigenstates) $\psi(r, t)=$ $\sum_{k} a_{k}(t)\left\langle r \mid \phi_{k}(t)\right\rangle e^{-i \int_{0}^{t} E_{k}\left(t^{\prime}\right) d t^{\prime}}$ into the radial Schrödinger equation provides a set of coupled differential equations. Integrating formally for $a_{k}(t)$ and assuming that for zeroth order $a_{k}^{(0)}(t)=\delta_{0, k}$, we get for first order [30,31]

$$
\begin{aligned}
a_{1}^{(1)}(t) & =-\int_{0}^{t} d t^{\prime}\left\langle\phi_{1}\left(t^{\prime}\right) \mid \dot{\phi}_{0}\left(t^{\prime}\right)\right\rangle e^{-\frac{i}{\hbar} \int_{0}^{t^{\prime}} d t^{\prime \prime}\left[E_{0}\left(t^{\prime \prime}\right)-E_{1}\left(t^{\prime \prime}\right)\right]} \\
& =-\int_{0}^{t} d t^{\prime} \frac{\dot{\omega}_{R}\left(t^{\prime}\right)}{2 \omega_{R}\left(t^{\prime}\right)} e^{2 i \int_{0}^{t^{\prime}} d t^{\prime \prime} \omega_{R}\left(t^{\prime \prime}\right)}
\end{aligned}
$$

where the second line follows by applying, in addition, the harmonic approximation. All other first-order amplitudes (for $k \geqslant$ 2 ) are zero, so the radial fidelity at $t_{f}$ can be calculated in this approximation as $F_{R}\left(t_{f}\right)=\left[1-\left|a_{1}^{(1)}\left(t_{f}\right)\right|^{2}\right]^{1 / 2}$. This is, in fact, correct up to second order, and it provides good agreement with the exact results, as shown by the symbols in the inset of Fig. 4.

\section{FULL 3D ANALYSIS}

Finally, we study numerically the actual coupled dynamics driven by the exact full potential, Eq. (3), combining all the effects considered so far and the longitudinal-radial coupling. The 3D fidelities are also compared with simpler 1D fidelities.

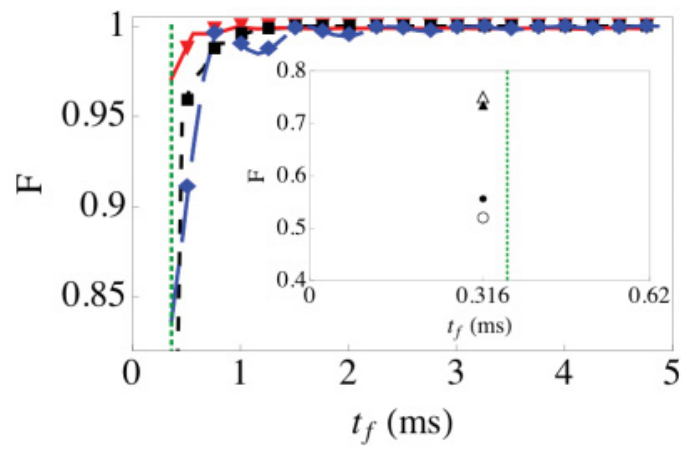

FIG. 7. (Color online) Fidelity for the $3 \mathrm{D}$ ground state vs different final times. Parameters: $v=0, \omega_{0 z} / 2 \pi=2500 \mathrm{~Hz}, \lambda=1060 \mathrm{~nm}$, and $\omega_{f z} / 2 \pi=250 \mathrm{~Hz}$. Protocols: inverse engineering, quintic $b$, $w_{0}=10 \mu \mathrm{m}$ (red solid line), and radial fidelity (red solid triangles); inverse engineering, quintic $b, w_{0}=3 \mu \mathrm{m}$ (black short-dashed line), and radial fidelity (solid squares); bang bang, $w_{0}=10 \mu \mathrm{m}$ (open circle in inset), and radial fidelity (solid circle); bang bang, $w_{0}=$ $3 \mu \mathrm{m}$ (open triangle in inset), and radial fidelity (solid triangle in inset); fast adiabatic, $w_{0}=3 \mu \mathrm{m}$, and $w_{0}=10 \mu \mathrm{m}$ (blue long-dashed line), and longitudinal fidelity (blue diamonds). The green vertical line marks the threshold for positive trap frequencies in the inverse engineering, quintic- $b$ protocol.

Before discussing the results a remark on the technicalities of the numerical calculations of the time-dependent wave functions is in order. To solve the longitudinal time-dependent Schrödinger equation we approximate the evolution operator $U_{L}$ using the split-operator method (SOM) [32] and fast Fourier transform (FFT) [33,34]. For the radial timedependent Schrödinger equation, we have used instead the finite-difference Crank-Nicholson scheme [34]. In this way the singular point $r=0$ can be excluded, imposing a zero of the wave function there for all $t$. For the 3D calculation we use similarly the Crank-Nicholson scheme with operator splitting for multidimensions (the radial and longitudinal directions) [34].

We calculate, starting from the ground state of the initial trap for $v=0$, the fidelity of the final state with respect to the ground state of the final trap using the three expansion protocols. Figure 7 clearly shows that the inverse-engineering protocol provides the best overall performance, with a fidelity decay at smaller times that can be avoided by increasing the waist. The radial-longitudinal quartic coupling does not play any significant role for the parameters of the example, and in any case it may also be suppressed by a waist increase.

As for the simple bang-bang approach, it fails in three dimensions, as expected, due to its poor radial behavior. (The radial fidelity alone is close to the $3 \mathrm{D}$ fidelity.) The results for fast adiabatic and inverse-engineering methods are not too different from each other for the chosen expansion, but the fast adiabatic method will fail sooner for more demanding expansions with smaller final frequencies, as clearly illustrated in Fig. 2(b), due to its inability to remain really adiabatic for the longitudinal direction. The main limitation of the inverse-engineering method is instead the possible failure of adiabaticity in the tighter radial direction, so it is intrinsically more robust than the fast adiabatic one. Note that the 3D fidelity is mimicked accurately by the $1 \mathrm{D}$ radial fidelity for 
inverse engineering and by the 1D longitudinal fidelity for the fast adiabatic approach.

\section{DISCUSSION AND OUTLOOK}

In this work we have analyzed the practical implementation of a transitionless expansion in a simple Gaussian optical dipole trap, taking into account anharmonicities, radiallongitudinal couplings, and the radial-longitudinal frequency mismatch. Three different protocols for expansions have been examined, which previously had been implemented in very different settings. We have compared their properties in this common physical implementation in a Gaussian optical dipole trap, and we have shown how to control difficulties due to three-dimensional effects.

The main conclusion of the study is that the transitionless expansions in optical traps are feasible under realistic conditions. Despite the relation between the longitudinal and transversal trapping frequencies through the intensity, the different time scales enable us to design fast expansions with high fidelities with respect to the ideal results using the invariant-based inverse-engineering method, which is particularly suitable compared to the two other approaches examined. Our detailed analysis of radial and longitudinal motions reveals the weakest points of each approach: for the inverse engineering, the main perturbation is due to the possible adiabaticity failure in the radial direction, which can be suppressed or mitigated by increasing the laser waist. This waist increase would also reduce smaller perturbing effects due to longitudinal anharmonicity or radial-longitudinal coupling. The simple bang-bang approach fails because the time for the radial expansion is badly mismatched with respect to the ideal time, and the fast adiabatic method fails at short times because of the adiabaticity failure in the longitudinal direction.

Complications such as perturbations due to different noise types and consideration of condensates, gravity effects, and the transient realization of imaginary trap frequencies are left for separate works. Other extensions of the present analysis could involve the addition of a second laser for further control of the potential shape or alternative traps. Optical traps based on Bessel laser beams, for example, may be interesting to decouple longitudinal and radial motions. Transport protocols are also amenable to a similar 3D analysis. Finally, the combination of invariant-based inverse engineering with optimal control theory to optimize the frequency design is also a promising venue of research [12,28].

\section{ACKNOWLEDGMENTS}

We thank G. C. Hegerfeldt for useful discussions. We acknowledge funding by the Basque government (Grant No. IT472-10), Ministerio de Ciencia e Innovación (Grant No. FIS2009-12773-C02-01), and the UPV/EHU under program UFI 11/55. X.C. thanks the Juan de la Cierva Programme, the National Natural Science Foundation of China (Grants No. 60806041 and No. 61176118), and the Shanghai Leading Academic Discipline Program (Grant No. S30105); E.T. thanks the Basque government (Grant No. BFI08.151); and D.G.O. thanks the Agence National de la Recherche, the Région Midi-Pyrénées, University Paul Sabatier (OMASYC project), and the Institut Universitaire de France.

\section{APPENDIX A: PERTURBATIVE TREATMENT OF LONGITUDINAL ANHARMONICITY}

To quantify the effect of anharmonicity in the inverseengineering method let us first expand the longitudinal Hamiltonian retaining the quartic term,

$$
\begin{aligned}
V(z) & =-\frac{V_{0}(t)}{1+z^{2} / z_{R}^{2}}+V_{0}(t) \approx V_{0} \frac{z^{2}}{z_{R}^{2}}-V_{0} \frac{z^{4}}{z_{R}^{4}} \\
& =\underbrace{\frac{1}{2} m \omega_{z}^{2}(t) z^{2}}_{:=V_{u}} \underbrace{-\frac{1}{2} m \omega_{z}^{2}(t) \frac{z^{4}}{z_{R}^{2}}}_{:=V_{1}} .
\end{aligned}
$$

The harmonic term is the unperturbed potential, and the quartic term is the perturbation. A further simplification to evaluate the fidelities $F_{L}=\left|\left\langle Z_{n}\left(t_{f}\right)\left|U_{z}\left(t_{f}, 0\right)\right| Z_{n}(0)\right\rangle\right|$ is to substitute the initial and final exact eigenstates $\left|Z_{n}(0)\right\rangle$ and $\left|Z_{n}\left(t_{f}\right)\right\rangle$ by corresponding eigenstates of the harmonic oscillator $\left|\psi_{n}(0)\right\rangle$ and $\left|\psi_{n}\left(t_{f}\right)\right\rangle$; see the expressions below. This is an excellent approximation for the lower eigenstates. In Fig. 3, for example, the overlap probability between exact and approximate states is 0.997 in the worst possible case ( $n=5, w_{0}=3 \mu \mathrm{m}$, and $\omega_{f z}=25 \mathrm{~Hz}$ ). In other words, the perturbation does not imply any significant deformation in the initial and final states. Its potential impact is instead at transient times where the energy may be high.

We thus expand the overlap $\left\langle\psi_{n}\left(t_{f}\right)\left|U_{L}\left(t_{f}, 0\right)\right| \psi_{n}(0)\right\rangle$ as $1+$ $f_{n, n}^{(1)}+f_{n, n}^{(2)}+\cdots$ in powers of $V_{1}$ to write

$$
F_{L}=\left|1+f_{n, n}^{(1)}+f_{n, n}^{(2)}+\cdots\right| .
$$

The first-order correction is given by

$$
\begin{aligned}
f_{n, n}^{(1)} & =\frac{-i}{\hbar} \int_{0}^{t_{f}} d t^{\prime}\left\langle\psi_{n}\left(t^{\prime}\right)\left|V_{1}\left(t^{\prime}\right)\right| \psi_{n}\left(t^{\prime}\right)\right\rangle \\
& =\frac{i m}{2 \hbar z_{R}^{2}} \int_{0}^{t_{f}} d t^{\prime} \omega_{L}^{2}\left(t^{\prime}\right)\left\langle\psi_{n}\left(t^{\prime}\right)\left|z^{4}\right| \psi_{n}\left(t^{\prime}\right)\right\rangle,
\end{aligned}
$$

and the unperturbed wave function corresponds to the known harmonic evolution of an expanding mode under the timedependent frequency $\omega_{z}(t)$ from an eigenstate of the initial harmonic oscillator [8],

$$
\begin{aligned}
\left\langle z \mid \psi_{n}\left(t^{\prime}\right)\right\rangle= & \frac{e^{\frac{i m}{\hbar} \frac{b_{z}{ }^{2}}{2 b}}}{\left(b 2^{n} n !\right)^{1 / 2}} e^{-i(n+1 / 2) \omega_{0 z} \int_{0}^{t^{\prime}} \frac{d t^{\prime \prime}}{b^{2}\left(t^{\prime \prime}\right)}} \\
& \times\left(\frac{m \omega_{0 z}}{\pi \hbar}\right)^{1 / 4} e^{-\frac{m \omega_{0} z^{2}}{2 \hbar b^{2}}} H_{n}\left(\sqrt{\frac{m \omega_{0 z}}{\hbar}} \frac{z}{b}\right) .
\end{aligned}
$$

This is nothing but the eigenvector of the quadratic invariant times the Lewis-Riesenfeld phase factor.

Using the triangular inequality $|x+y| \geqslant|| x|-| y||, F_{L} \geqslant$ $1-\left|f_{n, n}^{(1)}+f_{n, n}^{(2)}+\cdots\right|$ and assuming that the perturbative corrections satisfy $\left|f_{n, n}^{(1)}\right|>>\left|f_{n, n}^{(2)}\right|$, then

$$
F_{L} \geqslant 1-\left|f_{n, n}^{(1)}\right| \text {. }
$$

The relevant matrix element in Eq. (A3) can be calculated explicitly,

$$
\left\langle\psi_{n}\left(t^{\prime}\right)\left|z^{4}\right| \psi_{n}\left(t^{\prime}\right)\right\rangle=\left(\frac{\hbar}{m \omega_{0 z}}\right)^{2} \frac{3 b^{4}}{4}\left[(n+1)^{2}+n^{2}\right] .
$$


Using this result and the Ermakov equation,

$$
\left|f_{n, n}^{(1)}\right|=\frac{3 \hbar}{8 m z_{R}^{2}}\left[(n+1)^{2}+n^{2}\right]\left(t_{f}-\frac{1}{\omega_{0 z}^{2}} \int_{0}^{t_{f}} \ddot{b} b^{3} d t\right) .
$$

To bound $-\int_{0}^{t_{f}} \ddot{b} b^{3} d t$ subjected to the imposed constraints on $b$ at $t=0$ and $t_{f}$ we follow closely the method applied in [5] to bound the average energy. We integrate first by parts applying $\dot{b}(0)=\dot{b}\left(t_{f}\right)=0$ and minimize the resulting positive integral $\int_{0}^{t_{f}} b^{2} \dot{b}^{2} d t$, using the Euler-Lagrange equation $\ddot{b} b+\dot{b}^{2}=0$ for $b(0)=1, b\left(t_{f}\right)=\gamma$. These two conditions are fulfilled for a set of functions larger than the subset of functions that satisfy all boundary conditions in Eq. (12), so the minimization sets a lower bound for the later subset. The solution is $b=$ $\left[t\left(\gamma^{2}-1\right) / t_{f}+1\right]^{1 / 2}$, and with it the integral becomes

$$
\int_{0}^{t_{f}} b^{2} \dot{b}^{2} d t=\frac{\left(\gamma^{2}-1\right)^{2}}{4 t_{f}} .
$$

This $b$ is not as such a physically valid solution, as the derivatives would jump at the edges, but it maximizes the fidelity lower bound,

$$
F_{L} \geqslant 1-\left\{\frac{3 \hbar \lambda^{2}}{8 m \pi^{2} w_{0}^{4}}\left[(n+1)^{2}+n^{2}\right]\left[t_{f}+\frac{3\left(\gamma^{2}-1\right)^{2}}{4 t_{f} \omega_{0 z}^{2}}\right]\right\} .
$$

Take note that there is an optimal $t_{f}$ value and that the fidelity deteriorates when increasing the quantum number and improves by increasing the waist.

Using for $b$ the quintic polynomial in Eq. (13), we get, instead of Eq. (A8),

$$
\int_{0}^{t_{f}} b^{2} \dot{b}^{2} d t=\frac{10\left(\gamma^{2}-1\right)^{2}}{24871 t_{f}}\left(1101+1351 \gamma+1101 \gamma^{2}\right) .
$$

This tends to $0.44 \gamma^{4} / t_{f}$ for $\gamma \gg 1$, which, up to the constant factor, is the same dependence found for the optimal function.

The first-order analysis provides analytical results and some qualitative guidance, but for more accurate results we should resort to a second-order calculation. Instead of Eq. (A2) we may also write the fidelity for the $n$th state in terms of the corresponding probability as $F_{L}=\left(1-\sum_{n \neq n^{\prime}} P_{n^{\prime}}\right)^{1 / 2}$, where $P_{n^{\prime}}$ is the probability to find the system in the $n^{\prime}$ th state at $t_{f}$. This is approximated to second order using first-order nondiagonal terms,

$$
F_{L}=\sqrt{1-\sum_{n \neq n^{\prime}}\left|f_{n, n^{\prime}}^{(1)}\right|^{2}}
$$

where

$$
\begin{aligned}
f_{n, n^{\prime}}^{(1)} & =\frac{-i}{\hbar} \int_{0}^{t_{f}} d t^{\prime}\left\langle\psi_{n}\left(t^{\prime}\right)\left|V_{1}\left(t^{\prime}\right)\right| \psi_{n^{\prime}}\left(t^{\prime}\right)\right\rangle \\
& =\frac{i \hbar \lambda^{2}}{2 \pi w^{2} m w_{0}^{4} \omega_{0 z}^{2}} \frac{\alpha_{n, n^{\prime}} \beta_{n, n^{\prime}}(t)}{\sqrt{\pi 2^{n+n^{\prime}} n ! n^{\prime} !}}, \\
\alpha_{n, n^{\prime}} & =\int_{-\infty}^{\infty} d y e^{-y^{2}} H_{n}(y) H_{n^{\prime}}(y) y^{4}
\end{aligned}
$$

and

$$
\beta_{n, n^{\prime}}(t)=\int_{0}^{t} d t_{1} b^{4}\left(t_{1}\right) \omega_{z}^{2}\left(t_{1}\right) e^{-i\left(n^{\prime}-n\right) \omega_{0 z} \int_{0}^{t_{1}} \frac{d t_{2}}{b^{2}\left(t_{2}\right)}} .
$$

\section{APPENDIX B: THE CONDITION OF ADIABATICITY}

Consider a time-dependent Hamiltonian $\hat{H}_{0}(t)$, with instantaneous eigenstates and energies given by

$$
\hat{H}_{0}(t)|i(t)\rangle=E_{i}(t)|i(t)\rangle .
$$

The adiabaticity condition is given by [30]

$$
\left|\left\langle i(t) \mid \partial_{t} j(t)\right\rangle\right| \ll \frac{1}{\hbar}\left|E_{i}(t)-E_{j}(t)\right|, \quad i \neq j .
$$

Now we apply this general expression to get the adiabaticity condition on the longitudinal and radial frequencies of the Gaussian-beam trap. In the harmonic approximation the longitudinal Hamiltonian is given by Eq. (20),

$$
H_{\mathrm{har}}(z, t)=-\frac{\hbar^{2}}{2 m} \frac{\partial^{2}}{\partial z^{2}}+\frac{m \omega_{z}^{2}(t) z^{2}}{2},
$$

whereas the radial Hamiltonian is given, according to Eqs. (22), (23), and (24), by

$$
H_{\mathrm{har}}(r, t)=-\frac{\hbar^{2}}{2 m} \frac{\partial^{2}}{\partial r^{2}}+\frac{m \omega_{R}^{2}(t) r^{2}}{2}+\frac{\hbar^{2}}{2 m}\left(\frac{v^{2}-1 / 4}{r^{2}}\right) .
$$

The instantaneous eigenstates and energies of the Hamiltonian, Eq. (B3), are

$$
\begin{aligned}
\langle z \mid n(t)\rangle & =\left(\frac{m \omega_{z}}{\pi \hbar}\right)^{1 / 4} \sqrt{\frac{1}{2^{n} n !}} e^{-\frac{-m \omega_{z} z^{2}}{2 \hbar}} H_{n}\left(\sqrt{\frac{m \omega_{z}}{\hbar} z}\right), \\
E_{n}(t) & =\left(n+\frac{1}{2}\right) \hbar \omega_{z}, \quad n=0,1, \ldots,
\end{aligned}
$$

where $\omega_{z}=\omega_{z}(t)$ and $H_{n}$ is the Hermite polynomial. To get the adiabaticity condition for the ground state $i=0$ of the longitudinal Hamiltonian we insert these expressions for the eigenstates and energies in Eq. (B2). The overlap of the ground state with the first excited state $j=1$ is 0 , so the first nonvanishing overlap of the ground state is with the $j=2$ state. The adiabatic condition is satisfied if

$$
\frac{\sqrt{2} \dot{\omega}_{z}}{8 \omega_{z}^{2}} \ll 1
$$

For the radial Hamiltonian, Eq. (B4), the instantaneous eigenstates and energies for $v=0$ are [35]

$$
\begin{aligned}
\langle r \mid k(t)\rangle & =\sqrt{\frac{2 m \omega_{R} r}{\hbar}} e^{-\frac{-m \omega_{R} r^{2}}{2 \hbar}} L_{k}^{0}\left(\frac{m \omega_{R}}{\hbar} r^{2}\right), \\
E_{k}(t) & =(2 k+1) \hbar \omega_{R}, \quad k=0,1, \ldots,
\end{aligned}
$$

with $k$ being the radial quantum number and $L_{k}^{0}$ being the generalized Laguerre polynomial. Again $\omega_{R}=\omega_{R}(t)$. To get the adiabaticity condition for the radial direction we insert these expressions for $i=0$ and $j=1$ in Eq. (B2),

$$
\frac{\dot{\omega}_{R}}{4 \omega_{R}^{2}} \ll 1
$$


[1] A. Kastberg, W. D. Phillips, S. L. Rolston, R. J. C. Spreeuw, and P. S. Jessen, Phys. Rev. Lett. 74, 1542 (1995).

[2] A. E. Leanhardt, T. A. Pasquini, M. Saba, A. Schirotzek, Y. Shin, D. Kielpinski, D. E. Pritchard, and W. Ketterle, Science 301, 1513 (2003).

[3] W. Hänsel, P. Hommelhoff, T. W. Hänsch, and J. Reichel, Nature (London) 413, 498 (2001).

[4] T. Kinoshita, T. Wenger, and D. S. Weiss, Phys. Rev. A 71, 011602 (2005).

[5] X. Chen and J. G. Muga, Phys. Rev. A 82, 053403 (2010).

[6] P. Salamon, K. H. Hoffmann, Y. Rezek, and R. Kosloff, PhysChemChemPhys 11, 1027 (2009).

[7] J. G. Muga, X. Chen, A. Ruschhaupt, and D. Guéry-Odelin, J. Phys. B 42, 241001 (2009).

[8] X. Chen, A. Ruschhaupt, S. Schmidt, A. del Campo, D. GuéryOdelin, and J. G. Muga, Phys. Rev. Lett. 104, 063002 (2010).

[9] J. G. Muga, X. Chen, S. Ibáñez, I. Lizuain, and A. Ruschhaupt, J. Phys. B 43, 085509 (2010).

[10] S. Masuda and K. Nakamura, Proc. R. Soc. A 466, 1135 (2010).

[11] J. F. Schaff, X. L. Song, P. Vignolo, and G. Labeyrie, Phys. Rev. A 82, 033430 (2010); 83, 059911(E) (2011).

[12] D. Stefanatos, J. Ruths, and J.-S. Li, Phys. Rev. A 82, 063422 (2010).

[13] S. Masuda and K. Nakamura, Phys. Rev. A 84, 043434 (2011).

[14] J. F. Schaff, X. L. Song, P. Capuzzi, P. Vignolo, and G. Labeyrie, Europhys. Lett. 93, 23001 (2011).

[15] J. F. Schaff, P. Capuzzi, G. Labeyrie, and P. Vignolo, New J. Phys. 13, 113017 (2011).

[16] B. Andresen, K. H. Hoffmann, J. Nulton, A. Tsirlin, and P. Salamon, Eur. J. Phys. 32, 827 (2011).

[17] Y. Li, L.-A. Wu, and Z.-D. Wang, Phys. Rev. A 83, 043804 (2011).
[18] A. del Campo, Phys. Rev. A 84, 031606(R) (2011).

[19] A. del Campo, Europhys. Lett. 96, 60005 (2011).

[20] X. Chen, E. Torrontegui, and J. G. Muga, Phys. Rev. A 83, 062116 (2011).

[21] S. Ibáñez, S. Martínez-Garaot, X. Chen, E. Torrontegui, and J. G. Muga, Phys. Rev. A 84, 023415 (2011).

[22] S. Choi, R. Onofrio, and B. Sundaram, Phys. Rev. A 84, 051601(R) (2011).

[23] M. Barrett, J. Sauer, and M. S. Chapman, Phys. Rev. Lett. 87, 010404 (2001).

[24] G. L. Gattobigio, A. Couvert, M. Jeppesen, R. Mathevet, and D. Guery-Odelin, Phys. Rev. A 80, 041605(R) (2009).

[25] Y. Takasu, K. Maki, K. Komori, T. Takano, K. Honda, M. Kumakura, T. Yabuzaki, and Y. Takahashi, Phys. Rev. Lett. 91, 040404 (2003).

[26] R. Grimm, M. Weidemüller, and T. B. Ovchinnikov, Adv. At. Mol. Opt. Phys. 42, 95 (2000).

[27] J. M. Vogels (private communication).

[28] X. Chen, E. Torrontegui, D. Stefanatos, J.-S. Li, and J. G. Muga, Phys. Rev. A 84, 043415 (2011).

[29] S. Nemoto, Appl. Opt. 29, 1940 (1990).

[30] L. I. Schiff, Quantum Mechanics (McGraw-Hill, New York, 1949).

[31] M. Demirplak and S. A. Rice, J. Phys. Chem. 107, 9937 (2003).

[32] E. Torrontegui, J. Echanobe, A. Ruschhaupt, D. Guéry-Odelin, and J. G. Muga, Phys. Rev. A 82, 043420 (2010).

[33] R. Kosloff, J. Phys. Chem. 92, 2087 (1988).

[34] W. H. Press, S. A. Teukolsky, W. T. Vetterling, and B. P. Flanery, Numerical Recipes: The Art of Scientific Computing, 3rd ed. (Cambridge University Press, Cambridge, 2007).

[35] P. Camiz A. Gerardi, C. Marchioro, E. Presutti, and E. Scacciatelli, J. Math. Phys. 12, 2040 (1971). 